\title{
PROCESSOS DE APRENDIZAGEM EM EQUIPE DE PROJETO QUE UTILIZA METODOLOGIA ÁGIL
}

\author{
LEARNING PROCESSES IN A PROJECT TEAM USING AGILE METHODOLOGY
}

\author{
Eduarda Vieira Floriani \\ Mestre em Engenharia e Gestão do Conhecimento \\ Universidade Federal de Santa Catarina - UFSC \\ Florianópolis, $\mathrm{SC}-$ Brasil. \\ florianieduarda@gmail.com \\ Andrea Valéria Steil \\ Doutora em Engenharia de Produção \\ Universidade Federal de Santa Catarina - UFSC \\ Florianópolis, $\mathrm{SC}$ - Brasil. \\ andrea.steil@ufsc.ufsc.br
}

\section{Resumo}

Organizações de Tecnologia da Informação (TI) que utilizam metodologia ágil dependem dos processos de aprendizagem de suas equipes de projeto, uma vez que são elas que desenvolvem os produtos e serviços da organização. Compreender como ocorre o processo de aprendizagem de equipes de projeto que utilizam metodologia ágil em contexto real pode auxiliar no desenvolvimento de procedimentos para o aumento da taxa de sucesso de projetos. Este artigo tem como objetivo descrever como ocorre o processo de aprendizagem de uma equipe de projeto de uma grande organização de TI do sul do Brasil que utiliza a metodologia ágil na execução de seus projetos. A perspectiva teórica utilizada foi a de processos de grupos, que considera a aprendizagem como o processo iterativo de comportamentos de reflexão-ação entre os membros da equipe durante a realização de seu trabalho. Realizou-se um estudo de caso a partir da observação sistemática de reuniões da equipe, os quais foram analisados por meio de análise temática. Dos 1027 trechos de falas categorizados como comportamentos de aprendizagem durante as reuniões, 64\% referem-se a comportamentos de reflexão e $36 \%$ a comportamentos de ação. A análise dos resultados indica que os comportamentos de reflexão promovem comportamentos de ação e que a interface entre os comportamentos de aprendizagem é retroalimentada. O reforço de determinados comportamentos ajuda os membros da equipe a compreenderem o que é esperado e quais decisões são mais adequadas. Conclui-se que a metodologia ágil auxilia na composição de um ambiente de aprendizagem e, por fim, apresenta-se uma proposta de pesquisas futuras sobre o tema investigado.

Palavras-chave: Aprendizagem de equipes. Equipes de projetos. Metodologia ágil. Tecnologia da Informação. Processo de Aprendizagem. Ágil.

\begin{abstract}
Information Technology (IT) organizations that use agile methodology depend on the learning processes of their project teams, since they are the ones that facilitate the effective development of the organization's products and services. Understanding how the learning process of project teams using agile methodology in a real context occurs can help in the development of procedures to increase the project success rate. This article aims to describe how the learning process of a project team of a large IT organization in southern Brazil occurs that uses the agile methodology in the execution of its projects. The theoretical perspective used was team processes, which considers learning as the iterative process of reflection-action behaviors among team members during the performance of their work. A case study was prepared based on the systematic observation of team meetings, which were analyzed through thematic analysis. Of the 1027 sections of speech categorized as learning behaviors
\end{abstract}


during meetings, $64 \%$ refer to reflection behaviors and $36 \%$ to action behaviors. The results analysis indicates that reflection behaviors promote action behaviors and that the interface between learning behaviors is continuous. Reinforcing certain behaviors helps team members to understand what is expected and which decisions are most appropriate. It is concluded that the agile methodology helps in the composition of a learning environment and, finally, a proposal for future research on the investigated theme is presented.

Keywords: Team learning. Project teams. Agile methodology. Information Technology. Learning process. Agile.

\section{Cite como - American Psychological Association (APA)}

Floriani, E. V., \& Steil, A. V. (2021, Ed. Esp. jan./abr.). Processos de aprendizagem em equipe de projeto que utiliza metodologia ágil. Revista de Gestao e Projetos (GeP), 12(1), 149-171.

https://doi.org/10.5585/gep.v12i1.18750.

\section{Introdução}

Para lidar com inovações tecnológicas e mercados globalizados, o trabalho tem sido cada vez mais organizado em equipes de projeto, especialmente em organizações de Tecnologia da Informação (TI). Isto porque a estruturação por projetos possibilita maior adaptabilidade ao ambiente de trabalho complexo e à execução de tarefas dinâmicas (Veelen \& Ufkes, 2019).

As equipes são importantes instâncias de aprendizagem (Lehmann-Willenbrock, 2017). Nas organizações de TI, a aprendizagem de equipes tem sido crescentemente investigada (Edmondson, 1999; Kandukuri \& Nasina, 2017; Harvey, Bresman, Edmondson, \& Pisano, 2020) porque ela gera capacidade de resposta organizacional à mudança e vantagens em longo prazo (Edmondson, 2002). Por exemplo, existem evidências de que a aprendizagem da equipe diminui o custo de implementação de mudanças, aumenta a velocidade de entrega de produtos, a eficácia de processos operacionais (Akgün, Lynn, Keskin, \& Dogan, 2014) e o desempenho da equipe (Veelen \& Ufkes, 2019; Wiese \& Burke, 2019). Nas equipes de projeto, a aprendizagem gera maior agilidade na identificação de problemas e soluções, bem como auxilia na redução dos custos de planejamento, projeto e implementação (Kandukuri \& Nasina, 2017).

A perspectiva de aprendizagem de equipe como um processo de grupo de Edmondson, Dillon e Roloff (2007) descreve e mensura os comportamentos de aprendizagem da equipe durante a realização de suas atividades, ao invés de inferir que a aprendizagem aconteceu a partir de resultados pré-determinados. Os comportamentos de aprendizado são relevantes em ambientes de trabalho com ritmo acelerado, como as empresas de TI (Edmondson, 1999; Kandukuri $\&$ Nasina, 2017). Para que uma equipe realize 
mudanças e avance, os seus membros devem discutir premissas e opiniões divergentes abertamente, no âmbito da própria equipe, ao invés de privadamente ou fora da equipe. Essas atividades interativas são definidas como comportamentos de aprendizagem, pois é por meio dessas atividades que a aprendizagem ocorre no nível da equipe. Essas atividades possibilitam que as equipes detectem mudanças no ambiente, aprendam sobre as exigências dos clientes, melhorem a compreensão coletiva dos membros sobre uma situação, ou descubram consequências inesperadas de suas ações anteriores (Edmondson, 1999).

Em organizações de TI, projetos frequentemente são norteados por uma metodologia de gerenciamento. Um modelo muito utilizado é a metodologia ágil, uma abordagem com maior ênfase em indivíduos e interações do que em processos e ferramentas, e em flexibilização a mudanças do que documentação completa (Henriksen \& Pedersen, 2017; Khalil \& Kotaiah, 2017; Rasnacis \& Berzisa, 2017). No desenvolvimento ágil de software, a maior parte do tempo da equipe é usada para a execução, ao invés de planejamento (Serrador \& Pinto, 2015), há intenso compartilhamento de conhecimento face-a-face, confiança e trabalho intensivo em equipe (Kuusinen et al., 2017).

Mesmo que em pequeno número, existem pesquisas sobre o impacto da metodologia ágil na aprendizagem de equipes de projeto (Annosi, Hemphälä, \& Brunetta, 2018), mas há poucos estudos sobre como ocorre esse aprendizado (Harvey et al., 2020). A literatura disponível tem focado sua atenção em fatores como risco de projeto (Heravi \& Gholami, 2018) ou estilos de liderança (Zhang, Cao, \& Wang, 2018). Há, portanto, necessidade de estudos sobre processos de aprendizagem de equipes de projeto que utilizam metodologia ágil.

O objetivo deste artigo é descrever como ocorre o processo de aprendizagem de uma equipe de projeto de uma grande organização de TI do sul do Brasil que utiliza a metodologia ágil na execução de seus projetos. O método utilizado é qualitativo, com base na observação das reuniões diárias e quinzenais da equipe. As interações da equipe durante as reuniões foram gravadas, transcritas e categorizadas por meio da técnica de análise temática. $\mathrm{O}$ estudo apresenta as categorias e a frequência dos comportamentos de aprendizagem utilizados durante as reuniões, interpreta esses comportamentos no contexto do método ágil e apresenta recomendações para a aprendizagem contínua de equipes de projeto em organizações de TI.

A baixa taxa de sucesso de projetos em organizações de TI tem sido uma preocupação frequente de pesquisadores e é considerada uma das questões mais urgentes do contexto (Engelbrecht, Johnston, \& Hooper, 2017; Hidding \& Nicholas, 2017). O entendimento de como ocorre a aprendizagem de equipes de projetos pode auxiliar a lidar com desafios, 
como complexidade do projeto, diversidade da equipe, membros temporários, limites fluídos de equipe e infraestrutura organizacional (Edmondson \& Nembhard, 2009). Ao conhecer o processo de aprendizagem, gestores de organizações de TI poderão desenvolver políticas e práticas para o incentivo de comportamentos de aprendizagem de equipe ágil. Além desta seção introdutória, este artigo é composto por outras quatro seções: referencial teórico, procedimentos metodológicos, análise e discussão dos resultados e conclusões.

\section{Referencial teórico}

Nesta seção são apresentados os principais conceitos que servem de base para compreender a aprendizagem de equipes de projetos.

\subsection{Equipes de projetos}

Equipes são entidades que moldam e reforçam os comportamentos desejáveis de seus membros ao longo do tempo(Maruping \& Magni, 2015). Suas principais características são: 1) existência de dois ou mais indivíduos; 2) interação social entre seus membros; 3) realização de tarefas organizacionais relevantes; 4) ações baseadas em um ou mais objetivos comuns; 5) interdependência nas tarefas, que se reflete no fluxo do trabalho, nas metas e/ou nos resultados da equipe; 6) existência de papéis e responsabilidades diferentes; e 7) incorporação em um ambiente organizacional que estabelece limites e influencia as trocas e interações internas e externas dos seus membros (Kozlowski \& Bell, 2001).

As equipes são um meio de articulação entre os indivíduos e a organização, cujo intuito é solucionar problemas, promover a aprendizagem e realizar as reflexões necessárias para que a organização alcance seus objetivos. Kozlowski e Ilgen (2006) definem seis categorias de equipe: de produção, de serviço, de gestão, de projeto, de ação e realização e consultiva. Esta pesquisa se concentra em equipes de projeto, que realizam ações especializadas com restrição de tempo.

Uma equipe de projeto, formada por pessoas com diferentes conhecimentos, habilidades e experiências, precisa trabalhar de forma integrada (Koskinen \& Pihlanto, 2008). $\mathrm{O}$ seu processo de desenvolvimento requer adaptabilidade e capacidade de mudança para responder aos sinais do ambiente. Essa capacidade envolve a aprendizagem da equipe. Por constituir o mecanismo pelo qual as empresas aprendem (Edmondson, 2002), a equipe apresenta-se como unidade fundamental de aprendizagem na organização (Harvey et al., 2020).

\subsection{Aprendizagem de equipes}

Três áreas têm contribuído com informações sobre como equipes aprendem (Edmondson et al., 2007), gerando diferentes perspectivas acerca do que seja aprendizagem de equipe. Embora compreendam a 
aprendizagem de equipes de forma particular, essas três perspectivas compartilham um pressuposto comum: o de que coletivos, e não apenas indivíduos, podem aprender.

A primeira perspectiva possui suas bases metodológicas e intelectuais nos processos de manufatura e serviços de operações, com foco em curvas de aprendizagem (Wright, 1936; Adler, 1990). A segunda tem o aporte conceitual na psicologia social realizada em laboratório e busca identificar como membros de grupos pequenos coordenam suas ações e conhecimentos para o alcance de tarefas interdependentes (Liang, Moreland, \& Argote, 1995; Moreland \& Myaskovsky, 2000). Por fim, a terceira possui suas bases no comportamento organizacional micro, ou seja, nos processos psicossociais e processos de grupo. As duas primeiras perspectivas definem aprendizagem de equipe a partir de seus resultados, seja por meio do aumento da curva de aprendizagem, seja pelo alcance de um padrão pré-especificado de desempenho. A terceira perspectiva, por outro lado, compreende a aprendizagem de equipes a partir dos processos que ocorrem durante a realização do trabalho da equipe (Edmondson, 1999; Sarin \& McDermott, 2003).

Neste estudo, a aprendizagem é analisada na perspectiva de processos. As pesquisas nesta perspectiva têm crescido, o que demonstra o interesse em compreender como a aprendizagem é afetada por fatores contextuais (por exemplo: clima, objetivos e identidade da equipe) e como ela afeta o desempenho da equipe (Harvey et al., 2020). A perspectiva de aprendizagem de equipe como um processo define a aprendizagem como os comportamentos dos membros durante as atividades da equipe, ao invés de inferir que a aprendizagem ocorreu a partir de melhorias de desempenho ou resultados observados (Edmondson et al., 2007).

A equipe será capaz, mais facilmente, de apontar lacunas em seus planos e desenvolver mudanças, se os membros da equipe tiverem liberdade de fazer perguntas, experimentar, buscar feedback, refletir sobre resultados e discutir resultados inesperados abertamente, ao invés de privadamente ou fora do grupo (Koeslag-Kreunen, Van den Bossche; Hoven, Van der Klink \& Gijselaers, 2018). Estas atividades compõem o comportamento de aprendizagem e é por meio dele que a aprendizagem é encenada no nível do grupo (Edmondson, 1999).

Os comportamentos de aprendizagem, por sua vez, podem ser compreendidos como um processo iterativo de reflexão e ação. Esse processo enfatiza a necessidade de interação dos membros da equipe em tarefas interdependentes, as quais geram comportamentos de aprendizagem do tipo reflexão ou ação, que contribuem para o alcance das metas da equipe (Edmondson, 1999; 2002). A epistemologia por trás da ênfase na reflexão e ação visualiza o conhecimento e a aprendizagem na prática social. Portanto, a aprendizagem advém e é 
fundamentada por meio de práticas variadas de membros da equipe (Edmondson, 2002).

\section{O comportamento de reflexão} contribui para a criação de novos insights na equipe, os quais emergem da procura de acordos entre os membros. Exemplos de comportamentos de reflexão são dividir informação, pedir ajuda, procurar feedback, e falar sobre erros e problemas. $\mathrm{O}$ comportamento de ação põe em prática os novos insights e orienta a equipe em uma direção produtiva. Exemplos de comportamentos de ação são as mudanças de ação, as melhorias no desempenho, a tomada de decisões, os planos de ação, a solução de problemas e a experimentação. A equipe obtém e processa dados por meio dos comportamentos de reflexão-ação, que possibilitam que a equipe se adapte e melhore, o que configura o processo de aprendizagem de equipe (Edmondson, 1999).

Se não incentivados, os membros da equipe não se envolvem automaticamente em comportamentos de aprendizagem de equipe.

Cada comportamento de aprendizagem implica em correr um risco. Por exemplo, o compartilhamento de ideias torna as pessoas vulneráveis, assim como a co-construção requer coragem para mudar protocolos conhecidos e buscar controvérsia (KoeslagKreunen et al., 2018). Cada vez mais a aprendizagem precisa ser estimulada na prática. Os comportamentos de aprendizagem surgem e evoluem quando os membros da equipe interagem ao longo do tempo dentro de um contexto temporal e social. Por essência, o aprendizado de equipe é um fenômeno temporal (Lehmann-Willenbrock, 2017), onde os membros da equipe exploram diferentes perspectivas, fazem perguntas, desafiam suposições, examinam resultados inesperados e identificam falhas dentro de um processo construtivo de conflito. À medida que essas diferenças geram sentido e são integradas em um relato coerente, os membros da equipe constroem concordância mútua e significado (Edmondson et al., 2007).

A construção de significado, por meio de comportamentos de aprendizagem, é relevante em ambientes de trabalho com ritmo acelerado (Edmondson, 1999; Kandukuri \& Nasina, 2017). O contexto de trabalho da equipe de projeto deste estudo é uma organização de TI, que se caracteriza como um ambiente com ritmo de trabalho acelerado, onde os membros de equipes de projeto concluem suas tarefas como uma unidade coletiva e são gerenciados por metodologias ágeis de projetos. A equipe investigada age em condições complexas que necessitam de coordenação de tarefas em tempo real e improvisação de ações para solucionar problemas (Edmondson et al., 2007). A metodologia ágil norteia os rituais da equipe e influencia os comportamentos de aprendizagem.

\subsection{Metodologia ágil}

Os projetos são uma forma de organização flexível, restrita em recursos e 
temporária para desenvolver serviços, produtos ou demais resultados. Os projetos possuem alta importância e visibilidade dentro do contexto organizacional (Davis, 2017), com seus investimentos chegando a trilhões de dólares anualmente (Serrador \& Pinto, 2015). O gerenciamento eficaz de projetos de TI é fundamental, especialmente em função das interações entre os membros da equipe.

Uma forma de gerenciamento de projetos que visa governar os processos de trabalho em equipe é a metodologia ágil (Serrador \& Pinto, 2015). Esta metodologia promove a distribuição de funções $\mathrm{e}$ responsabilidades na equipe, incentiva a comunicação frequente entre clientes e membros da equipe, e divide o trabalho em tarefas e entregas regulares. Há clamores de que essas características sejam positivas para o gerenciamento de equipes de desenvolvimento de software (Noguera, Guerrero-Roldán, \& Masó, 2017) e estimulem a aprendizagem da mesma, no entanto, é algo ainda pouco explorado/evidenciado em estudos científicos.

O objetivo da equipe que trabalha com metodologia ágil é elaborar continuamente versões estáveis e aprimoradas do produto, com foco em atender às necessidades do cliente. A equipe ágil prepara um plano de suas atividades, por meio da seleção de tarefas a serem implementadas e do cálculo de custo, tempo e esforço necessários para a tarefa selecionada. No final de cada tarefa, é feita uma revisão do que foi desenvolvido, onde os membros da equipe julgam se o desenvolvimento está de acordo com as necessidades. Neste processo, os membros da equipe ágil podem encontrar determinado problema e tomar medidas corretivas para solucioná-lo (Anwer, Aftab, Shah, \& Waheed, 2017).

Apesar de possuir características e objetivos similares, a metodologia ágil possui uma diversidade de formatos. Na equipe pesquisada, as metodologias ágeis utilizadas são Kanban e Scrum. A equipe utiliza o método de visualização do Kanban para auxiliar na visualização de todo o trabalho que será desenvolvido, está em desenvolvimento, ou já foi finalizado. Em relação ao Scrum, a equipe utiliza as reuniões diárias e as reuniões de retrospectiva. A reunião diária ajuda os membros da equipe a conhecer o andamento do projeto. A retrospectiva é a reunião onde os membros da equipe discutem o que está funcionando e o que não está funcionando no processo. Isso ajuda a decidir quais práticas devem ser realizadas e o que deve ser alterado na continuidade do projeto (Anwer et al., 2017).

\section{Procedimentos metodológicos e considerações éticas}

Esta seção apresenta os procedimentos aplicados e descreve os sujeitos de pesquisa selecionados para atender ao objetivo da pesquisa. $\mathrm{O}$ projeto desta pesquisa foi submetido ao Sistema Nacional de Ética em Pesquisa e aprovado (número CAAE 
13203819.4.0000.0121) antes do início da coleta de dados.

\subsection{Delineamento metodológico}

O método de pesquisa escolhido foi o estudo de caso único, qualitativo e descritivo, tendo como nível de análise a equipe de projeto. Neste estudo, os pesquisadores buscaram informações provenientes de diversas fontes de dados (entrevistas, observações, documentos), analisaram as características de uma determinada amostra e interpretaram as categorias temáticas de interesse que ajudaram a compreender o fenômeno aprendizagem da equipe de projeto (Merriam \& Tisdell, 2016). O estudo de caso é apropriado porque se busca uma compreensão profunda do contexto. O interesse está no percurso e não nos resultados, na descoberta e não na confirmação, no contexto e não em uma variável específica (Merriam \& Tisdell, 2016).

\subsection{Participantes}

O foco deste estudo é uma equipe de desenvolvimento de software de uma organização de TI, que faz parte do polo tecnológico do sul do país. A escolha por este setor se deu pelo fato dos estudos na área de TI ainda serem incipientes quando comparados com a sua relevância econômica e social. O ramo de TI é um dos que mais cria empregos e cresce no país, ocupando posição central na criação e no aperfeiçoamento da infraestrutura e inovação tecnológica (Singh \& Hess, 2017).
Além disso, o setor de TI mostra-se um meio dinâmico e intensivo em conhecimento, onde utilizam-se ativos intangíveis para a condução de negócios, como recursos de informação e conhecimento (Soto-Acosta, Popa, \& Martinez-Conesa, 2018).

A organização que abriga a equipe é de grande porte, com mais de 500 funcionários e aproximadamente 10 anos de mercado. A organização não é puramente baseada em projetos, visto que tem em sua estrutura o trabalho em torno de projetos e também trabalhos focados em especializações funcionais. A escolha dessa organização levou em conta fatores de acessibilidade $\mathrm{e}$ conveniência (Vergara, 2004). Os critérios para seleção da equipe foram: trabalhar em um projeto com nível de avanço de cerca de $70 \%$, operar com um prazo específico para finalização de atividades e utilizar metodologia ágil de projetos. A equipe selecionada contava com nove pessoas em cinco cargos: um coordenador de engenharia (CE), cinco desenvolvedores de software (DS), um gerente de produto (GR), um analista de qualidade (AQ) e um designer de interação (DI). A identidade da organização e dos participantes foi preservada.

Dentre os entrevistados, oito não estavam em cargos de liderança (gerente de produto, desenvolvedores, designer de interação e analista de qualidade) e um era coordenador de engenharia. Apesar da nomenclatura do cargo, o gerente de produto não exerce papel de liderança em seu escopo 
de atuação. Portanto, apenas um dos entrevistados tinha responsabilidade de gestão de pessoas dentro da equipe. O coordenador de engenharia liderava os desenvolvedores e o analista de qualidade da equipe de projeto e outra equipe, que não fazia parte da equipe de projeto desta pesquisa. $\mathrm{O}$ gerente de produto e o designer de interação eram liderados por outras pessoas, que não faziam parte da equipe de projeto deste estudo. A Figura 1 sumariza a hierarquia da equipe.

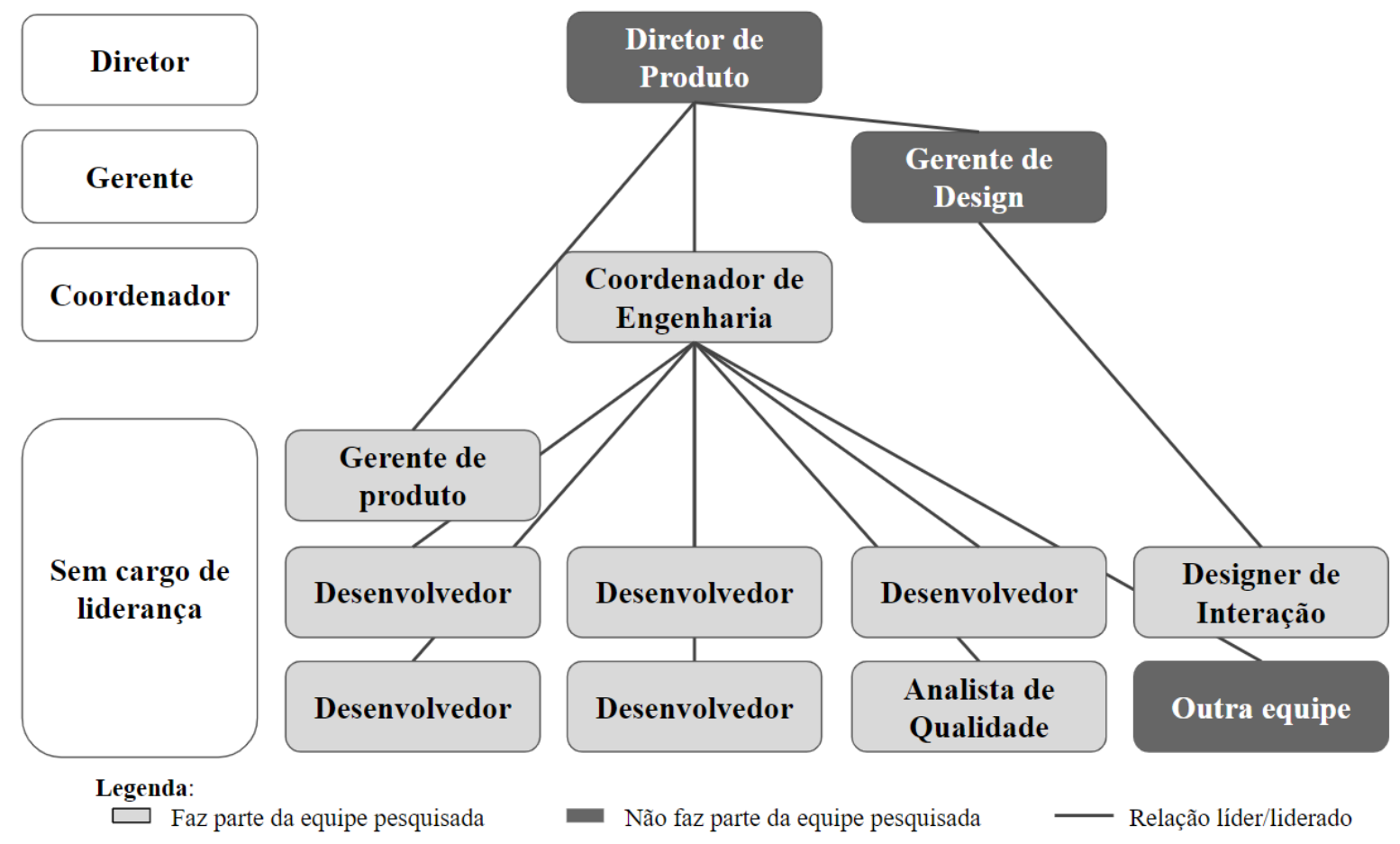

Figura 1. Organograma da equipe de projeto estudada Fonte: Elaborada pelas autoras (2020).

\subsection{Coleta de dados}

Esta pesquisa teve um enfoque transversal, onde os dados coletados são relativos a um determinado período no tempo (Fink, 2009). Os dados foram coletados entre agosto e setembro de 2019. A estrutura e o funcionamento da equipe possibilitaram a realização de entrevistas e observação de reuniões com seus membros, em diferentes períodos de tempo. Para complementar as fontes de evidências, também foram consultados documentos, como relatórios e atas de reuniões.

Apesar de utilizar múltiplas fontes de informação (Taylor \& Bogdan, 1994; Merriam \& Tisdell, 2016), a principal fonte foi a observação de 13 (treze) reuniões da equipe. Destas, dez foram reuniões diárias e três foram reuniões de retrospectiva (que acontecem a cada quinze dias). A reunião diária costuma durar menos do que 30 minutos e é utilizada para repassar o que cada membro está fazendo e definir as prioridades de entrega do projeto. 
A reunião de retrospectiva tem duração média de uma hora e trinta minutos e é por meio dela que a equipe discute as entregas, as métricas, os pontos negativos e os pontos positivos da quinzena. A reunião de retrospectiva não exclui a reunião diária.

A observação das reuniões contou com um protocolo de indagação (Taylor \& Bogdan, 1994), com os seguintes eixos: características das reuniões, características de interação da equipe, características de ação da equipe, características de reflexão da equipe e comentários gerais. Todas as reuniões foram gravadas e transcritas, com o consentimento prévio dos entrevistados. O tempo de duração das reuniões foi em média 30 minutos, que resultou em 142 páginas de texto transcrito. A próxima seção detalha o processo de análise dos dados.

\subsection{Procedimentos de análise de dados}

Os dados foram analisados por meio de análise temática, que consiste no processo de identificação, organização e oferecimento de insights sobre padrões de significado de dados qualitativos. Nesta pesquisa, os dados qualitativos objeto da análise temática são as transcrições das comunicações dos membros da equipe de projeto durante as reuniões diárias e de retrospectiva.

Utilizou-se predominantemente a análise temática dedutiva, na qual as pesquisadoras partem de um modelo ou referencial teórico para guiar o processo de codificação e interpretação dos dados (Braun \& Clarke, 2012). As pesquisadoras também ficaram atentas à emergência de novos códigos dos dados (análise temática indutiva).

A perspectiva teórica deste estudo foi a de processos de equipes, que considera a aprendizagem como o processo iterativo de comportamentos de reflexão e de ação entre os membros da equipe. Os comportamentos de aprendizagem de reflexão são aqueles que colaboram para a criação de novos insights na equipe, já os comportamentos de aprendizagem de ação são aqueles que levam à prática os novos insights e orientam a equipe em uma direção produtiva (Edmondson et al., 2007). Ao todo, foram pré-definidas duas categorias temáticas (comportamentos de aprendizagem de reflexão e comportamentos de aprendizagem de ação), 15 subcategorias e 28 códigos para guiar a interpretação e a análise dos dados.

Durante a análise de dados, as subcategorias e os códigos sofreram alterações em função do caráter cíclico da análise. Nesse processo se constrói o sentido dos dados, que implica em mudanças na codificação e nas categorias temáticas (Braun \& Clarke, 2012). A Figura 2 exemplifica o processo de análise temática. 


\section{Trecho de fala}

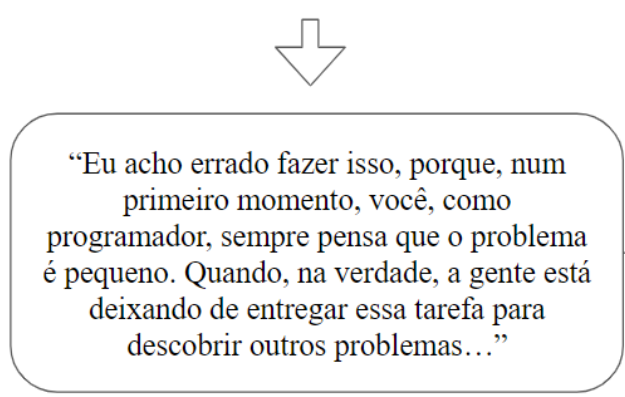

"Qual o nosso desafio? No mínimo tentar manter essa previsibilidade, então tentar se comportar, repetir comportamentos para que a gente mantenha a previsibilidade ou, no melhor cenário, tentar diminuir a previsão também. Sabendo que a gente pode negociar escopo, mas não qualidade."

\section{Código}

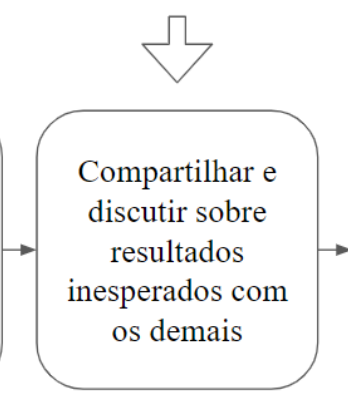

Subcategorização

Categorização
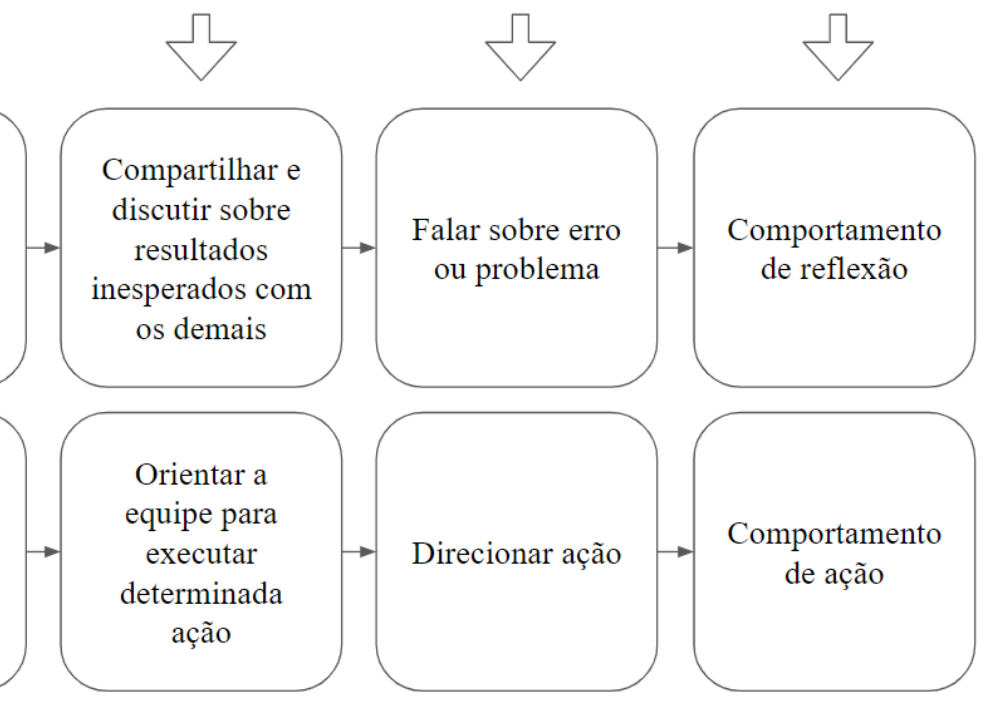

Figura 2. Exemplificação do processo de análise temática com citações das reuniões observadas

Fonte: Elaborado pelas autoras (2020).

Foram identificadas as seguintes subcategorias com base na literatura (Edmondson, 1999): compartilhar percepção, falar sobre erros e problemas, procurar feedback, pedir ajuda, tomar decisão, compartilhar melhorias no desempenho, efetuar mudanças, realizar experimentação, estruturar planos de ação e solucionar problemas. Além destas subcategorias, outras emergiram dos dados: direcionar ação, fazer questionamentos, tirar dúvida, estimular a percepção e oferecer ajuda. Destaca-se que a delimitação das categorias, subcategorias e códigos possui finalidade pedagógica e descritiva para a compreensão do fenômeno de interesse, mas não pretende sugerir linearidade ou etapas sequenciais de seus componentes. Pelo contrário, essas categorias estão estreitamente conectadas e interligadas.
O processo de análise dos dados evoluiu de acordo com ciclos de codificação, caracterizado por métodos específicos (Saldaña, 2015).

\section{Análise e discussão dos resultados}

Nesta seção, os resultados são apresentados e discutidos, com a finalidade de compreender como ocorre a aprendizagem de equipe baseada nos comportamentos de ação e reflexão durante a realização do projeto.

\subsection{Resultados}

Os comportamentos de aprendizagem de reflexão e os comportamentos de aprendizagem de ação foram identificados a partir de 1027 trechos de falas transcritos, dos quais 656 foram relacionados a comportamentos de reflexão (64\%) e 371 a comportamentos de ação (36\%). Na Tabela 1 
observa-se a quantidade de trechos de fala das oito subcategorias e 16 códigos dos comportamentos de aprendizagem de reflexão. As oito subcategorias dos comportamentos de reflexão são: compartilhar percepção $(30 \%, \mathrm{n}$ $=194)$, falar sobre erros e problemas $(22 \%, \mathrm{n}$
= 143), tirar dúvida $(19 \%, \mathrm{n}=125)$, estimular a percepção $(17 \%, \mathrm{n}=109)$, fazer questionamentos $(9 \%, \mathrm{n}=58)$, procurar feedback $(3 \%, \mathrm{n}=19)$, oferecer ajuda $(1 \%, \mathrm{n}=$ 6) e pedir ajuda $(0,3 \%, \mathrm{n}=2)$.

Tabela 1 - Representação da quantidade de categorias, subcategorias e códigos coletados

\begin{tabular}{|c|c|c|c|c|c|}
\hline Categorização & Qtde & Subcategorização & Qtde & Códigos & Qtde \\
\hline \multirow{16}{*}{$\begin{array}{l}\text { Comportamentos } \\
\text { de aprendizagem } \\
\text { de reflexão }\end{array}$} & \multirow{16}{*}{656} & \multirow{2}{*}{$\begin{array}{l}\text { Fazer } \\
\text { questionamentos }\end{array}$} & \multirow{2}{*}{58} & $\begin{array}{l}\text { Buscar compreender melhor, por meio de } \\
\text { perguntas diretivas ou questões reflexivas, o } \\
\text { motivo de determinada linha de raciocínio }\end{array}$ & 43 \\
\hline & & & & $\begin{array}{l}\text { Buscar compreender melhor, por meio de } \\
\text { perguntas diretivas ou questões reflexivas, o } \\
\text { motivo de determinada decisão }\end{array}$ & 15 \\
\hline & & \multirow{2}{*}{ Procurar feedback } & \multirow{2}{*}{19} & $\begin{array}{l}\text { Buscar o retorno de terceiros para uma decisão } \\
\text { própria }\end{array}$ & 17 \\
\hline & & & & $\begin{array}{l}\text { Buscar o retorno de terceiros sobre um } \\
\text { comportamento próprio }\end{array}$ & 2 \\
\hline & & $\begin{array}{l}\text { Compartilhar } \\
\text { percepção }\end{array}$ & 194 & Explicitar a percepção sobre determinado assunto & 194 \\
\hline & & Pedir ajuda & 2 & Solicitar auxílio para realizar algo & 2 \\
\hline & & \multirow{3}{*}{ Tirar dúvida } & \multirow{3}{*}{125} & Tirar dúvida para executar determinada atividade & 20 \\
\hline & & & & $\begin{array}{l}\text { Tirar dúvida para construir uma linha de } \\
\text { raciocínio }\end{array}$ & 76 \\
\hline & & & & Tirar dúvida para tomar uma decisão & 29 \\
\hline & & \multirow{3}{*}{$\begin{array}{l}\text { Estimular a } \\
\text { percepção }\end{array}$} & \multirow{3}{*}{109} & $\begin{array}{l}\text { Estimular que a equipe se ajude ao executar } \\
\text { determinada atividade }\end{array}$ & 14 \\
\hline & & & & $\begin{array}{l}\text { Estimular que a equipe construa uma linha de } \\
\text { raciocínio }\end{array}$ & 24 \\
\hline & & & & Estimular que a equipe tome uma decisão & 71 \\
\hline & & \multirow{2}{*}{ Oferecer ajuda } & \multirow{2}{*}{6} & $\begin{array}{l}\text { Questionar se um terceiro necessita de ajuda em } \\
\text { determinada atividade }\end{array}$ & 2 \\
\hline & & & & $\begin{array}{l}\text { Oferecer ajuda a um terceiro para executar } \\
\text { determinada atividade }\end{array}$ & 4 \\
\hline & & \multirow{2}{*}{$\begin{array}{l}\text { Falar sobre erro ou } \\
\text { problema }\end{array}$} & \multirow{2}{*}{143} & $\begin{array}{l}\text { Compartilhar e discutir sobre comportamentos } \\
\text { equivocados com os demais }\end{array}$ & 59 \\
\hline & & & & $\begin{array}{l}\text { Compartilhar e discutir sobre resultados } \\
\text { inesperados com os demais }\end{array}$ & 84 \\
\hline
\end{tabular}

Fonte: Elaborado pelas autoras (2020).

A Tabela 2 apresenta as sete subcategorias e 12 códigos dos comportamentos de aprendizagem de ação. As sete subcategorias dos comportamentos de aprendizagem baseados em ação são: direcionar ação $(57 \%, \mathrm{n}=210)$, tomar decisão 
$(28 \%, \mathrm{n}=103)$, compartilhar melhorias no desempenho $(6.5 \%, \mathrm{n}=24)$, efetuar mudanças $(4 \%, \mathrm{n}=16)$, realizar experimentação $(4 \%, \mathrm{n}=$
16), estruturar planos de ação $(0.5 \%, \mathrm{n}=2)$ e solucionar problemas $(0 \%, \mathrm{n}=0)$.

Tabela 2 - Representação da quantidade de categorias, subcategorias e códigos coletados

\begin{tabular}{|c|c|c|c|c|c|}
\hline Categorização & Qtde & Subcategorização & Qtde & Códigos & Qtde \\
\hline \multirow{12}{*}{$\begin{array}{l}\text { Comportamentos } \\
\text { de aprendizagem } \\
\text { de ação }\end{array}$} & \multirow{12}{*}{371} & \multirow{2}{*}{ Efetuar mudanças } & \multirow{2}{*}{16} & $\begin{array}{l}\text { Alterar a execução de determinada atividade, após } \\
\text { o seu planejamento }\end{array}$ & 2 \\
\hline & & & & $\begin{array}{l}\text { Falar sobre a mudança na execução de } \\
\text { determinada atividade, após o seu planejamento }\end{array}$ & 14 \\
\hline & & \multirow{2}{*}{$\begin{array}{l}\text { Compartilhar } \\
\text { melhorias no } \\
\text { desempenho }\end{array}$} & \multirow{2}{*}{24} & $\begin{array}{l}\text { Compartilhar evolução positiva na execução das } \\
\text { atividades }\end{array}$ & 17 \\
\hline & & & & $\begin{array}{l}\text { Compartilhar evolução positiva em } \\
\text { comportamento }\end{array}$ & 7 \\
\hline & & \multirow{2}{*}{ Tomar decisão } & \multirow{2}{*}{103} & Escolher uma opção dentre as alternativas & 81 \\
\hline & & & & Deliberar sobre determinado assunto & 22 \\
\hline & & $\begin{array}{l}\text { Estruturar planos } \\
\text { de ação }\end{array}$ & 2 & $\begin{array}{l}\text { Montar uma estrutura de acompanhamento de } \\
\text { atividades (atividades, prazos e responsáveis) para } \\
\text { atingimento de objetivos }\end{array}$ & 2 \\
\hline & & Direcionar ação & 210 & Orientar a equipe para executar determinada ação & 210 \\
\hline & & \multirow{2}{*}{$\begin{array}{l}\text { Solucionar } \\
\text { problemas }\end{array}$} & \multirow{2}{*}{0} & $\begin{array}{l}\text { Desenvolver mudanças para solucionar resultados } \\
\text { inesperados }\end{array}$ & 0 \\
\hline & & & & $\begin{array}{l}\text { Desenvolver mudanças para solucionar } \\
\text { comportamentos equivocados }\end{array}$ & 0 \\
\hline & & \multirow{2}{*}{$\begin{array}{l}\text { Realizar } \\
\text { experimentação }\end{array}$} & \multirow[b]{2}{*}{16} & Ato de experimentar coisas novas & 0 \\
\hline & & & & $\begin{array}{l}\text { Colocar em prática uma linha de raciocínio a fim } \\
\text { de verificar se está correta ou não }\end{array}$ & 16 \\
\hline
\end{tabular}

Fonte: Elaborado pelas autoras (2020).

Além da quantidade de comportamentos de reflexão e de ação, destaca-se a quantidade total de comportamentos de aprendizagem durante as reuniões diárias e de retrospectiva. 
Tabela 3. Representação da quantidade de comportamentos de aprendizagem de ação e de reflexão durante as reuniões diárias e de retrospectiva

\begin{tabular}{|c|c|c|c|c|}
\hline Reunião & Data fictícia & Ação & Reflexão & Total \\
\hline \multirow{10}{*}{ 쯤 } & 1 & 29 & 31 & 60 \\
\hline & 3 & 17 & 32 & 49 \\
\hline & 4 & 21 & 32 & 53 \\
\hline & 5 & 19 & 21 & 40 \\
\hline & 6 & 15 & 53 & 68 \\
\hline & 8 & 21 & 34 & 55 \\
\hline & 9 & 26 & 20 & 46 \\
\hline & 10 & 21 & 23 & 44 \\
\hline & 11 & 30 & 39 & 69 \\
\hline & 13 & 42 & 59 & 101 \\
\hline \multirow{3}{*}{$\begin{array}{l}\text { 옳 } \\
\text { ㅁ }\end{array}$} & 2 & 33 & 43 & 76 \\
\hline & 7 & 54 & 120 & 174 \\
\hline & 12 & 43 & 149 & 192 \\
\hline \multicolumn{2}{|c|}{ Total geral } & 371 & 656 & 1027 \\
\hline
\end{tabular}

Fonte: Elaborado pelas autoras (2020).

A Tabela 3 apresenta a frequência de comportamentos de aprendizagem dentro de cada reunião. A disposição das reuniões está por ordem cronológica, portanto, a primeira reunião foi a reunião diária com data fictícia número 1, já a segunda foi a reunião de retrospectiva descrita com o número 2 , e assim segue consecutivamente. Esses resultados serão discutidos na próxima seção.

\subsection{Discussão}

As interações entre os membros da equipe durante as reuniões diárias e de retrospectiva foram oportunidades para a apresentação de comportamentos de aprendizagem de reflexão e de ação, ilustrando que o contexto em que uma equipe atua influencia o seu aprendizado (Edmondson et al., 2007). Os membros da equipe que estão envolvidos em processos e atividades de aprendizagem fazem perguntas, exploram diferentes visões e perspectivas do problema, examinam todas as suposições, resultados possíveis e, consequentemente, mudam seu comportamento de aprendizagem ao longo do tempo (Lehmann-Willenbrock, 2017). Dos 1027 trechos de falas, os comportamentos de reflexão (64\%) tiveram maior representatividade do que os comportamentos de ação (36\%), o que significa que, dentro das reuniões de equipe, a aprendizagem através dos comportamentos de reflexão (ex: compartilhar percepção) é maior do que a 
aprendizagem através dos comportamentos de ação (ex: tomar decisão).

As duas subcategorias com maior prevalência dentre os comportamentos de aprendizagem foram direcionar ação (comportamento de ação, $\mathrm{n}=210$ ) e compartilhar percepção (comportamento de reflexão, $\mathrm{n}=194)$. $\mathrm{O}$ comportamento direcionar ação aparece significativamente em todas as reuniões da equipe, o que demonstra que a equipe necessita de comportamentos que a orientem a uma direção produtiva no encerramento das reuniões. O comportamento compartilhar percepção se refere ao ato de opinar sobre qual tarefa deve ser priorizada e quem deve ser o responsável por ela. No contexto desta equipe, esse comportamento contribuiu para a resolução de problemas identificados durante o desenvolvimento do projeto.

Subcategorias que também tiveram frequência significativa, com mais de 100 trechos de fala, foram: falar sobre erros e problemas (comportamento de reflexão, $\mathrm{n}=$ 143), tirar dúvida (comportamento de reflexão, $\mathrm{n}=125), \quad$ estimular a percepção (comportamento de reflexão, $\mathrm{n}=109$ ) e tomar decisão (comportamento de ação, $\mathrm{n}=103$ ). A alta frequência do comportamento falar sobre erros e problemas demonstra que os membros da equipe costumam reportar erros ao invés de escondê-los, o que colabora para a construção de uma equipe que aprende com suas falhas (Edmondson et al., 2007; Wiese \& Burke, 2019). Já a alta frequência em tirar dúvidas (reflexão, $\mathrm{n}=125$ ) sugere que os membros da equipe são estimulados a fazer perguntas e a sair das reuniões sem dúvidas. Diferentemente de tirar dúvidas, a subcategoria estimular a percepção não tem o intuito de responder algo que o locutor desconhece, e sim promover a reflexão entre os membros da equipe. $\mathrm{O}$ comportamento tomar decisão possui o número de falas mais bem distribuído entre a equipe, pois todos tiveram trechos de fala nesta subcategoria. O processo de tomada de decisão incentiva os membros a considerarem uma variedade de informações, contribuições e restrições e terem uma visão mais ampla de seus trabalhos (Sarin \& McDermott, 2003).

Dentre as subcategorias com poucos trechos de fala, temos: fazer questionamentos (reflexão, $\mathrm{n}=58$ ), compartilhar melhorias no desempenho (ação, $\mathrm{n}=24$ ), procurar feedback (reflexão, $n=19$ ), efetuar mudanças (ação, $n=$ 16), realizar experimentação (ação, $\mathrm{n}=16$ ), oferecer ajuda (reflexão, $\mathrm{n}=6$ ), pedir ajuda (reflexão, $\mathrm{n}=2$ ), estruturar planos de ação (ação, $n=2$ ) e solucionar problemas (ação, $n=$ $0)$. Surpreende que comportamentos associados à execução (como efetuar mudanças e realizar experimentação) não aparecem com frequência neste estudo. A expectativa era a de que esses comportamentos fossem mais expressivos, pois a equipe executava um projeto com nível de avanço de $70 \%$ e com um prazo de finalização próximo. Além disso, comportamentos de ação são valorizados na metodologia ágil, onde a maior parte do tempo da equipe é usada para a 
execução, ao invés de planejamento de atividades (Serrador \& Pinto, 2015).

Uma possível explicação para este fato se deve à coleta de dados ter sido dentro das reuniões da equipe e não no dia a dia do trabalho, o que leva à conclusão de que, provavelmente, as reuniões da equipe são o espaço seguro de reflexão da equipe antes da execução das tarefas rotineiras. Exemplos de subcategorias com baixa frequência possivelmente vinculadas e este motivo são: efetuar mudanças, realizar experimentação e solucionar problemas. Por mais que a equipe não tenha experimentado e desenvolvido soluções ao longo das reuniões, nas subcategorias 'falar sobre erros e problemas' e ‘compartilhar percepções' fez-se a reflexão acerca dos problemas, e na subcategoria 'direcionar ação' apresentaram-se possibilidades de resolução, que, provavelmente, serão realizadas após as reuniões da equipe.

Outro ponto que ajuda a explicar a baixa frequência de determinadas subcategorias, como procurar feedback, oferecer ajuda, pedir ajuda e estruturar planos de ação, é a singularidade dos comportamentos de reflexão e ação de uma equipe que utiliza metodologia ágil. Nesse contexto, determinadas práticas já são incorporadas na equipe sem a necessidade de explicitação. Por exemplo, o comportamento procurar feedback, como uma busca de retorno de terceiros para uma decisão própria, se mostra dispensável em um cenário onde a equipe, constantemente, recebe retorno sobre a execução de seus projetos por meio de outras subcategorias, como: compartilhar percepção ou tirar dúvida. Os comportamentos oferecer e pedir ajuda não tem frequência significativa, pois o trabalho cooperativo já faz parte da metodologia ágil os membros da equipe devem trabalhar diariamente em conjunto (Beck et al., 2001). Além disso, a metodologia ágil deixa subentendidas algumas regras de trabalho, como, por exemplo, que todas as tarefas delegadas nas reuniões diárias devem ser realizadas até o dia seguinte. Essa pode ser uma hipótese para o fato de o comportamento de estruturar planos de ação, com detalhamento de atividades, prazos e responsáveis, aparecer com pouca frequência.

Os achados demonstram que, por mais que os comportamentos de aprendizagem definidos como procurar feedback, oferecer ajuda, pedir ajuda e estruturar planos de ação não apareçam com frequência significativa no estudo, o processo de aprendizagem ocorreu. Estes comportamentos não foram observados por serem possivelmente desnecessários ao contexto e deram espaço para outros comportamentos de aprendizagem emergirem, como: direcionar ação, compartilhar percepção, falar sobre erros e problemas, tirar dúvida, estimular a percepção e tomar decisão.

A metodologia ágil facilita os comportamentos de ação denominados direcionar ação e tomar decisão, pois dá protagonismo às equipes ao promover uma distribuição de responsabilidades, incentivar a 
comunicação frequente entre clientes e membros da equipe, e dividir o trabalho em tarefas e entregas regulares (Noguera et al., 2017). O comportamento direcionar ação auxilia a equipe a compreender os objetivos da organização para atingir as metas de tempo, de satisfação do cliente e de resultados de negócio. Ao ter ciência dos objetivos organizacionais, os membros da equipe têm embasamento para a tomada de decisão (Cavazotte, Moreno, \& Turano, 2015), podendo exercer a autonomia em seu nível hierárquico.

Em relação aos comportamentos de reflexão, a metodologia ágil promove os comportamentos de compartilhar percepção, falar sobre erros e problemas, tirar dúvidas e estimular a percepção. Os princípios da metodologia ágil salientam que os membros da equipe devem reportar erros ao invés de escondê-los e trabalhar em conjunto ao invés de individualmente. Essas práticas colaboram para a construção de uma equipe que aprende com suas falhas (Wiese \& Burke, 2019). Os momentos de troca de conhecimento são relevantes para a construção da equipe por possibilitar que os membros reflitam sobre suas ações, gerem mudanças e se tornem uma unidade de trabalho integrada (Koskinen \& Pihlanto, 2008; Widmann, Messmann, \& Mulder, 2016).

O reforço de determinado comportamento ajuda os membros da equipe a compreenderem o que é esperado e quais decisões são mais adequadas. Os comportamentos de falar sobre erro ou problema e compartilhar melhorias de desempenho auxiliam nesse aspecto. Esses comportamentos foram observados com mais frequência nas reuniões de retrospectiva, por ser o espaço destinado ao compartilhamento de soluções para os problemas encontrados no trabalho (Annosi, Martini, Brunetta, \& Marchegiani, 2018). A reunião de retrospectiva é um ritual consolidado na metodologia ágil, que se mostrou importante para a livre troca de ideias e construção de conhecimento macro e multifuncional.

Embora evidencie-se neste estudo que a metodologia ágil se configura em um contexto condutivo aos comportamentos de aprendizagem, não se pode inferir que apenas com a utilização da metodologia ágil estes comportamentos serão observados. Por exemplo, na equipe pesquisada, a metodologia ágil não foi suficiente para viabilizar comportamentos como: experimentar coisas novas e buscar retorno de terceiros sobre um comportamento próprio. Uma explicação possível para a falta de trechos de fala em ato de experimentar coisas novas é que, muitas vezes, a pressão para executar o trabalho é maior (Annosi et al., 2018) do que a necessidade de criar novos processos e práticas. Dentre os demais comportamentos de aprendizagem, o ato de experimentar coisas novas é o que mais aproxima a equipe do conceito de inovação. Por mais que as equipes tenham valor potencial para inovação (Widmann et al., 2016), a responsabilidade dos 
membros da equipe pesquisada não consiste na inovação em si, e sim na codificação e/ou execução de atividades previamente estabelecidas.

A priorização da execução é reforçada quando o prazo de finalização do projeto está próximo. Neste cenário, o ato de procurar feedback sobre comportamentos não foi observado em quantidade significativa nas reuniões de equipe. Os incentivos de feedback estão voltados para decisões referentes à melhoria do produto ou serviço para o consumidor final e não para o aprimoramento profissional dos membros da equipe. Ao pensar na melhoria do produto, os membros da equipe ajustam tarefas e estratégias, mas não significa necessariamente que os mesmos reflitam sobre conhecimentos, habilidades ou atitudes que devam ser aprimoradas.
Os resultados deste estudo agregam evidências às proposições de Edmondson et al. (2007) ao identificar que comportamentos de reflexão promovem comportamentos de ação e que a relação entre os comportamentos de aprendizagem é retroalimentada. A Figura 3 explicita a interface dinâmica entre as duas categorias temáticas (comportamentos de aprendizagem baseados em reflexão e comportamentos de aprendizagem baseados na ação). Os trechos de fala representam o contexto de uma das reuniões da equipe e descrevem informações interpretadas como comportamentos de aprendizagem focados em reflexão ou ação. 
Floriani, E. V., \& Steil, A. V. (2021, Ed. Esp. jan./abr.). Processos de aprendizagem em equipe de projeto que utiliza metodologia ágil

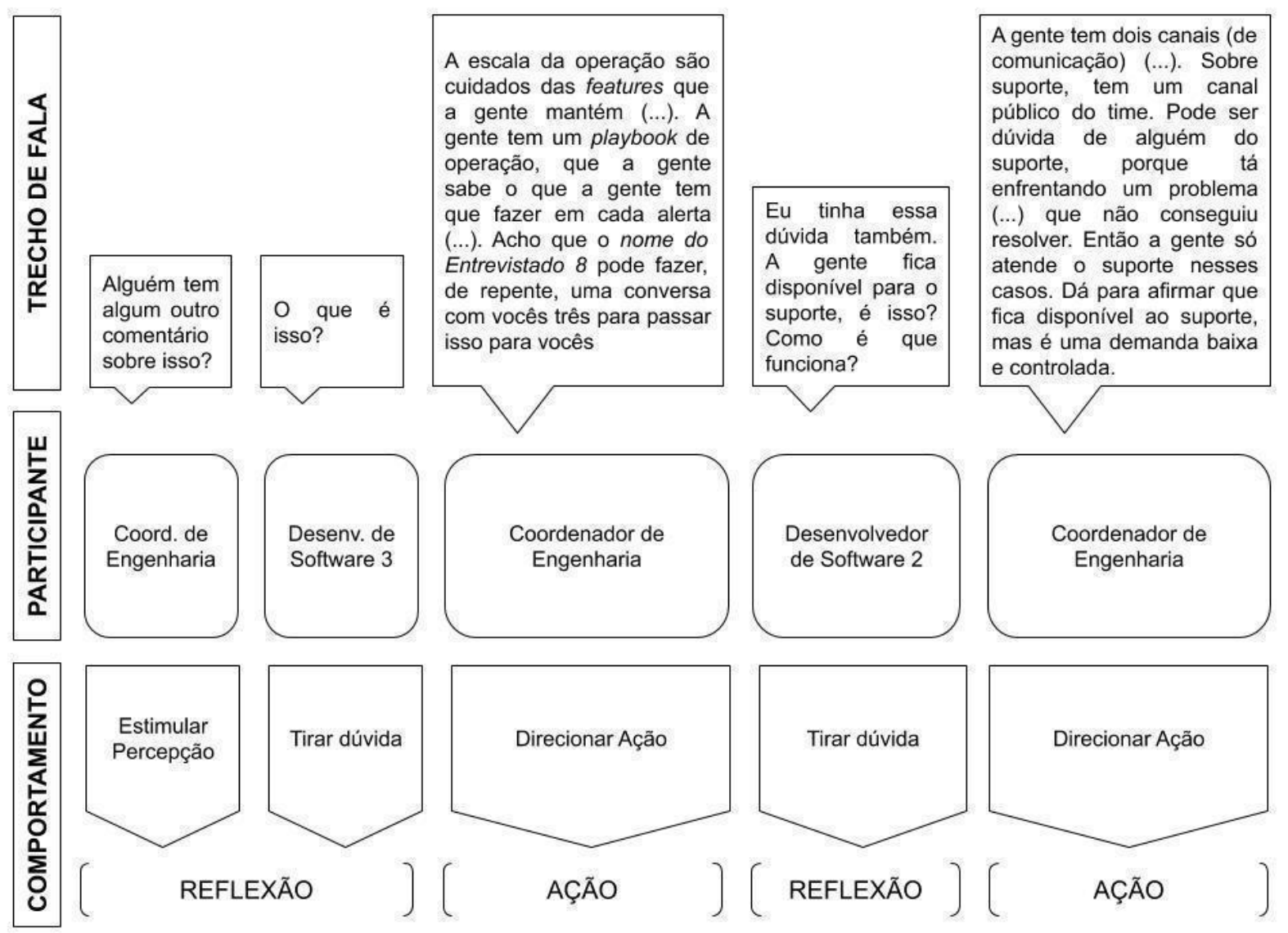

Figura 3. Falas que descrevem a interface dinâmica entre os comportamentos de reflexão e ação.

Fonte: Elaborado pelas autoras (2020).

$\mathrm{O}$ contexto em que uma equipe atua influencia o aprendizado da equipe por poder apresentar (ou impedir) a oportunidade de se envolver em comportamentos de aprendizagem (Edmondson et al., 2007). Se os membros não interagem e não compartilham conhecimento, a equipe não irá construir redes de conhecimento que contribuam para a resolução de problemas no desenvolvimento de produtos. As interações dos membros da equipe e o conhecimento sobre os saberes de cada membro auxiliam na criação e na troca de conhecimento (Akgün et al., 2014).

\section{Conclusões}

Este estudo apresenta a interface dos comportamentos de aprendizagem de reflexãoação no contexto de uma equipe de projetos que utiliza a metodologia ágil na execução de seus projetos. A aprendizagem dessa equipe ocorre por meio de comportamentos que são explicitados ao longo das reuniões da equipe (ex: direcionar ação, compartilhar percepção, etc.). Determinados comportamentos não necessitam ser explicitados, pois resultam de demais comportamentos de aprendizagem. Isso foi verificado no comportamento procurar 
feedback, onde o retorno sobre a execução de projetos é feito por meio de outras subcategorias como compartilhar percepção ou tirar dúvida. A metodologia ágil facilita a promoção de comportamentos de aprendizagem, mas não garante que estes aconteçam. Por exemplo, o ato de experimentar coisas novas e a busca de retorno de terceiros sobre um comportamento próprio são comportamentos, em tese, promovidos pelas práticas ágeis, mas que não foram observados no estudo. Cabe implementar a metodologia ágil dentro de ciclos pequenos de mudanças, com poucas alterações para possibilitar a identificação de melhorias e ajustes no processo.

Os resultados demonstram que, para a equipe pesquisada, é importante discutir as opiniões divergentes abertamente para encontrar lacunas em seus planos e realizar mudanças. A equipe pesquisada obtém e processa dados por meio dos comportamentos de reflexão e ação, com o objetivo de se adaptar e melhorar, o que caracteriza o processo de aprendizagem de equipe. Os resultados reforçam as evidências de estudos anteriores sobre aprendizagem de equipe como um processo de grupo, onde os processos de aprendizagem de equipe são detalhados sem se preocupar, necessariamente, com os resultados imediatos de avanço no projeto.

Este estudo contribui para o avanço do conhecimento sobre aprendizagem de equipes de projeto em duas frentes principais: (i) o aprofundamento da aprendizagem de equipe no contexto de TI, e (ii) o potencial de metodologias de gestão de projetos para auxiliar o processo de aprendizagem da equipe. As contribuições estão relacionadas ao objetivo da pesquisa: compreender como ocorre o processo de aprendizagem de equipe.

Apesar das contribuições da pesquisa, as escolhas metodológicas refletem em algumas limitações: i) o processo de análise temática é um processo de codificação norteado pelos dados, os padrões identificados nesta análise temática são válidos apenas para a equipe estudada; ii) a análise temática foi realizada com os dados coletados nas reuniões da equipe e não na rotina de trabalho; e, iii) em vias de delimitar o escopo da pesquisa, esta possui corte transversal, restrito a determinado período no tempo, o que impossibilita a análise longitudinal.

As limitações e os resultados da pesquisa abrem um campo de oportunidades de futuros estudos. A compreensão dos processos de aprendizagem, baseados em comportamentos de reflexão-ação, de uma equipe de projeto em outros contextos organizacionais seria interessante para analisar semelhanças, diferenças e padrões de comportamento de aprendizagem. Da mesma forma, diferentes delineamentos metodológicos possivelmente implicariam em diferentes resultados. Por exemplo, estudos longitudinais poderiam prover maiores informações sobre a aprendizagem de equipes de projetos em diferentes períodos de tempo, assim como, a coleta de dados por meio da 
rotina de trabalho da equipe de projeto e não apenas das reuniões de equipe poderiam implicar numa maior frequência de comportamentos de aprendizagem baseados em ação do que em reflexão. Por fim, cabe um maior número de estudos para compreender como a aprendizagem de equipe pode contribuir para a aprendizagem organizacional.

Assim, as oportunidades de aprendizagem da organização podem ser mais significativas durante o percurso do projeto, quando erros e problemas são analisados em tempo real.

\section{Referências}

Adler, P. S. (1990). Shared learning. Management Science, 36(8), 938-957. doi: 10.1287/mnsc.36.8.938

Akgün, A., Lynn, G. S., Keskin, H., \& Dogan, D. (2014). Team learning in IT implementation projects: antecedents and consequences. International Journal of Information Management, 34(1), 37-47. doi: 10.1016/j.ijinfomgt.2013.09.007

Annosi, M. C., Hemphälä, J., \& Brunetta, F. (2018). Investigating the impact of agile methods on learning and innovation. In: Boccardelli, P., Annosi, M. C., Brunetta, F., \& Magnusson, M. (eds) Learning and Innovation in Hybrid Organizations. Palgrave Macmillan, Cham. doi: 10.1007/978-3-319-62467-9_5

Annosi, M. C., Martini, A., Brunetta, F., \& Marchegiani, L. (2018). Learning in an agile setting: a multilevel research study on the evolution of organizational routines. Journal of Business Research, 1-13. doi: 10.1016/j.jbusres.2018.05.011

Anwer, F., Aftab, S., Shah, S.M., \& Waheed, U. (2017). Comparative analysis of two popular agile process models: extreme programming and scrum. International Journal of Computer Science and
Telecommunications, 8(2), 1-7. doi: 10.31838/ijccts/08.02.01

Beck, K. et al. Manifesto for agile software development, 2001. Disponível em: http: http://www.agilemanifesto.org. Acesso em: jun. 2020.

Braun, V., \& Clarke, V. (2012). Thematic analysis. In: Cooper, H., Camic, P. M., Long, D. L., Panter, A. T., Rindskopf, D., \& Sher, K. J. (Eds.). APA handbook of research methods in psychology. Washington, DC: American Psychological Association, 2, 57-71. doi: 10.1037/13620004

Cavazotte, F. S. C. N., Moreno, V. A., \& Turano, L. M. (2015). Cultura de aprendizagem contínua, atitudes e desempenho no trabalho: uma comparação entre empresas do setor público e privado. Revista de Administração Pública, 49(6), 1555-1578. doi: 10.1590/0034-7612136534.

Davis, K. (2017). An empirical investigation into different stakeholder groups perception of project success. International Journal of Project Management, 35(4), 604-617. doi: 10.1016/j.ijproman.2017.02.004

Edmondson, A. C. (1999). Psychological safety and learning behavior in work teams. Administrative Science Quarterly Science, 44(2), 350-383. doi: 10.2307/2666999

Edmondson, A. C. (2002) The local and variegated nature of learning in organizations: A group-level perspective. Organization Science, 13(2), 128-146. doi: 10.1287/orsc.13.2.128.530

Edmondson, A. C., Dillon, J. R., \& Roloff, K. S. (2007). Three perspectives on team learning. The Academy of Management Annals, 1(1), 269-314. doi: $10.1080 / 078559811$

Edmondson, A., \& Nembhard, I. (2009). Product development and learning in project teams: the challenges are the benefits. The Journal of Product Innovation Management, 
26, 123-138. doi: 10.1111/j.1540-

5885.2009.00341.x

Engelbrecht, J., Johnston, K. A., \& Hooper, V. (2017). The influence of business managers' IT competence on IT project success. International Journal of Project Management, 35(6), 994-1005. doi: 10.1016/j.ijproman.2017.04.016

Fink, A. (2009). How to conduct surveys: a step-by-step guide. Fourth Edition, London, UK: SAGE Publications Inc.

Harvey, J. F., Bresman, H., Edmondson, A. C., \& Pisano, G. (2020). Team learning and superior firm performance: a meso-level perspective on dynamic capabilities. Harvard Business School Technology \& Operations, Working Paper 19-059. doi: 10.5465/AMBPP.2018.18086abstract

Henriksen, A., \& Pedersen, S. A. (2017). A qualitative case study on agile practices and project success in agile software projects. Journal of Modern Project Management, 5(1), 62-73. doi: 10.19255/JMPM01306

Heravi, G., \& Gholami, A. (2018). The influence of project risk management maturity and organizational learning on the success of power plant construction projects. Project Management Journal, 49(5), 22-37. doi: $10.1177 / 8756972818786661$

Hidding, G. J., \& Nicholas, J. M. (2017). A new way of thinking about IT project management practices: early empirical results. Journal of Organizational Computing and Electronic Commerce, 27(1), 81-95. doi: 10.1080/10919392.2016.1263113

Kandukuri, V., \& Nasina, J. (2017). Collaborative team learning contributions to the knowledge management process, leadership, and culture in a multinational indian IT company. International Journal of Business and Information, 12(2). doi: 10.6702/ijbi.2017.12.2.1

Khalil, M. A., \& Kotaiah, B. (2017). Implementation of agile methodology based on SCRUM tool. 2017 International Conference on Energy, Communication, Data Analytics and Soft Computing (ICECDS), 2351-2357. doi: 10.1109/ICECDS.2017.8389872

Koeslag-Kreunen, M.; Van den Bossche, P.; Hoven, M.; Van der Klink, M.; \& Gijselaers, W. (2018). When leadership powers team learning: a meta analysis. Small Group Research, 49(4), 475-513. doi: $10.1177 / 1046496418764824$

Kozlowski, S. W. J., \& Bell, B. S. (2001). Work groups and teams in organizations. Cornell University, ILR School.

Kozlowski, S. W. J., \& Ilgen, D. R. (2006). Enhancing the effectiveness of work groups and teams. Psychological Science in the Public Interest, 7(3), 77-124. doi: 10.1111/j.1529-1006.2006.00030.x

Koskinen, K., \& Pihlanto, P. (2008). Knowledge management in project-based companies. New York: Palgrave Macmillan. doi: 10.1057/9780230595071

Kuusinen, K., Peggy, G., Sharp, H., Barroca, L., Taylor, K., \& Wood, L. (2017). Knowledge sharing in a large agile organisation: a survey study. In: 18th International Conference. Agile Processes in Software Engineering and Extreme Programming, 135-150. doi: 10.1007/978-3319-57633-6_9

Lehmann-Willenbrock, N. (2017). Team learning: new insights through a temporal lens. Small Group Research, 48(2), 123130. doi: $10.1177 / 1046496416689308$

Liang, D. W., Moreland, R. L., \& Argote, L. (1995). Group versus individual training and group performance: the mediating factor of transactive memory. Personality and Social Psychology Bulletin, 21(4), 384-393. doi: 10.1177/0146167295214009

Maruping, L. M., \& Magni, M. (2015). Motivating employees to explore collaboration technology in team contexts. Management Information Systems 
Quarterly, 39(1), 1-16. doi: $10.5902 / 1983465923853$

Merriam, S. B., \& Tisdell, E. J. (2016). Qualitative research. A guide to design and implementation. 4. ed. San Francisco: Jossey-Bass.

Moreland, R. L., \& Myaskovsky, L. (2000). Exploring the performance benefits of group training: Transactive memory or improved communication? Organizational Behavior and Human Decision Processes, 82(1), 117. doi: 10.1006/obhd.2000.2891

Noguera, I., Guerrero-Roldán, A. E., \& Masó, R. (2017). Collaborative agile learning in online environments: Strategies for improving team regulation and project management. Computers \& Education. doi: 10.1016/j.compedu.2017.09.008.

Rasnacis, A., \& Berzisa, S. (2017). Method for adaptation and implementation of agile project management methodology. Procedia Computer Science, 104, 43-50. doi: 10.1016/j.procs.2017.01.055

Sarin, S., \& McDermott, C. (2003). The effect of team leader characteristics on learning, knowledge application, and performance of cross-functional new product development teams. Decision Sciences, 34(4), 707-739. doi: 10.1111/j.1540-5414.2003.02350.x

Serrador, P., \& Pinto, J. K. (2015). Does Agile work? - A quantitative analysis of agile project success. International Journal of Project Management, 33(5), 1040-1051. doi: 10.1016/j.ijproman.2015.01.006

Singh, A. \& Hess, T. (2017). How chief digital officers promote the digital transformation of their companies. Management Information Systems Quarterly Executive, 16(1). doi: 10.4324/9780429286797-9

Soto-Acosta, P., Popa, S., \& Martinez-Conesa, I. (2018). Information technology, knowledge management and environmental dynamism as drivers of innovation ambidexterity: a study in SMEs. Journal of
Knowledge Management, 22(4), 824-849. doi: 10.1108/JKM-10-2017-0448

Taylor, S., \& Bogdan, R. (1994). Introducción a los métodos cualitativos. Buenos Aires: Paidos.

Veelen, R. V., \& Ufkes, E. G. (2019). Teaming up or down? A multisource study on the role of team identification and learning in the team diversity-performance link. Group \& Organization Management, 44(1), 38-71. doi: $10.1177 / 1059601117750532$

Vergara, S. C. (2004). Projetos e relatórios de pesquisa em administração. São Paulo: Atlas.

Widmann, A., Messmann, G., \& Mulder, R. H. (2016). The impact of team learning behaviors on team innovative work behavior: a systematic review. Human Resource Development Review, 15(4), 429458. doi: 10.1177/1534484316673713

Wiese, C. W., \& Burke, C. S. (2019). Understanding team learning dynamics over time. Frontiers in Psychology, 10(1417), 114. doi: 10.3389/fpsyg.2019.01417

Wright, T. P. (1936). Factors affecting the cost of airplanes. Journal of Aeronautical Sciences, 3(4), 122-128. doi: 10.2514/8.155

Zhang, L., Cao, T., \& Wang, Y. (2018). The mediation role of leadership styles in integrated project collaboration: An emotional intelligence perspective. International Journal of Project Management, 36(2), 317-330. doi: 10.1016/j.ijproman.2017.08.014. 